Stefan Braum

\title{
Rechtswissenschaft und Rechtskultur ${ }^{1}$
}

\section{Summary}

We are witnesses of an erosion. Algorithms appear to compose oneself, us, our daily lives to measure our lives. Not only intelligence services impinge on the core of privacy, collect data, store, relay and sell them. Personal profiles are "screened" for security risks and commercial exploitation. Both systems, such as capital and financial markets and institutions - NSA, BND, SREL, Facebook, and Google - often act independently of the law. Systems and institutions know more about us, than we do. More importantly, the legislator is driven by activism. What used to be rule yesterday is no longer valid today, and what seems to be mandatory today is arbitrary tomorrow. It is not a good time for the legal culture, whose definition gives only just ex negativo, because we know what we can not and will not perceive as belonging to the culture of law. And it is a difficult time for the jurisprudence. What can these accomplish, especially when taught and operated at the universities, what can it contribute to a European society, where normative integration and freedom are upheld? An attempted answer can only be outlined here.

\section{Résumé}

Nous sommes les témoins d'une érosion. Petit à petit, des algorithmes commencent à nous jauger - nous, notre quotidien, notre vie. Les services secrets ne sont pas les seuls à infiltrer notre vie privée, à collecter nos données, à les enregistrer, les transférer, les vendre. Les profils personnels sont passés au peigne fin, au nom de mesures sécuritaires ou de la commercialisation. Des systèmes tels que les marchés de capitaux et financiers, de même que des institutions - NSA, BND, SREL, Facebook et Google - franchissent eux aussi souvent les limites du cadre juridique et légal dans leurs agissements. Ces systèmes et institutions en savent plus sur nous que nous-mêmes. Le législateur est en outre mû par l'actionnisme. Ce qui avait force de loi hier n'est plus applicable aujourd'hui; ce qui semble contraignant aujourd'hui deviendra insignifiant demain. Ce n'est pas une époque propice à la culture juridique - qui ne se définit d'ailleurs qu'ex negativo, en ce sens que nous savons ce que nous ne pouvons et voulons considérer comme appartenant à la culture du droit. Et c'est une époque difficile pour les sciences juridiques. Que peuvent-elles apporter, surtout lorsqu'elles sont enseignées et pratiquées dans les universités, en quoi peuvent-elles contribuer à une société européenne attachée à une intégration et une liberté normatives? Nous ne pouvons qu'esquisser une tentative de réponse à cette question.

1 Vortrag gehalten bei der Jahrestagung der Luxemburgischen Anwaltskammer. Der Vortragsstil wird hier beibehalten. 


\section{Aufklärung - der Ruf nach der Rechtswissenschaft}

Alles beginnt mit einem Wagnis: Mache Gebrauch von deinem eigenen Verstand, fordert die politische Philosophie der Aufklärung. Gebrauche ihn, um dich aus Unfreiheit und Unmündigkeit zu befreien und sei dir gewiss, dass nichts Äußerliches wichtig ist. Zwei Dinge bleiben - a priori, für immer: das „moralische Gesetz in uns“ und der „,bestirnte Himmel über uns". Weil wenig von Dauer ist, bleibt uns nur die Kraft, die in der Freiheit liegt - das ist die Entdeckung der Aufklärung. Wir sind unhinterfragbar Menschen, deren Würde sich aus der gedachten Möglichkeit zur Freiheit ergibt. Diese Möglichkeit führen wir mit, sie muss als Kern unberührt bleiben, gerade dann, wenn wir uns als Bürger in einem Staat oder in über den Staat hinausweisenden Institutionen organisieren. Die Botschaft der politischen Philosophie der Aufklärung ist: ohne Freiheit kein Recht. Recht, das den legitimen Anspruch erheben will, angewendet und gegebenenfalls durch Zwang umgesetzt zu werden, ist der Freiheit verpflichtet - oder es ist kein Recht.

Die juristische Figur, die die politische Philosophie der Aufklärung einatmet, ist der Vertrag. Er folgt dem Prinzip der Privatautonomie. Zwei Parteien begründen ein Anerkennungsverhältnis, in dem sie sich verpflichten, die Freiheit des anderen wie die eigene zu achten und für die Schäden aufzukommen, die sie - schuldhaft - dem anderen zufügen. Der Vertrag ist das rechtliche Mittel des aufstrebenden Bürgertums des 18. und 19. Jahrhunderts. Er erlaubt freies wirtschaftliches Handeln, das sich von staatlichen Zwängen und Kontrollinteressen befreit und wird zum Ideal einer Rechtskultur der Freiheit. Die Rechtswissenschaft, die um den Vertrag entsteht, sichert Vertragsbegründung und Auslegung ab, sie entwickelt Methoden, fragt nach Wortsinn und Zweck einer Vereinbarung, hält die Parteien an ihrem erklärten Willen fest, berücksichtigt Irrtümer und Täuschungen. Ihr Gegenstand sind Transparenz und Berechenbarkeit, sie will die politisch errungenen Freiheitsrechte schützen und sie entwickelt Regeln für Sanktionen, die aus der Verletzung von Freiheitsrechten folgen müssen. Das ist idealistisch und sehr politisch zugleich, denn dieses Verständnis von Rechtswissenschaften als Teil einer Kultur der Freiheit richtet sich gegen autoritären Absolutismus, geheime Kabinettsjustiz und richterliche Willkür. Die Gesetzgebung des 18. und 19. Jahrhunderts ist durch die politische Philosophie der Freiheit geprägt. Verfassungen entstehen, der machtvolle Staat wird konstitutionell eingefasst und begrenzt. Die Mächtigen müssen sich in aller Offenheit legitimieren, indem sie Bürgerrechte anerkennen und schützen. Moderne Strafgesetze, die dem humanen Strafen verpflichtet sind, entstehen. Die Folter als Mittel des Strafverfahrens wird geächtet. Moderne Kodifikationen wie zum Beispiel der Code Napoléon oder das Bürgerliche Gesetzbuch erheben die Privatautonomie und den mündigen Bürger, der sein wirtschaftliches Glück selbst in die Hand nimmt, zum Prinzip.

\section{Rechtswissenschaft - im Dienste staatlicher Ordnung}

Ideale verändern leider nicht immer die Wirklichkeit, sondern die Wirklichkeit verändert die Ideale. Das Versprechen der Aufklärung - Freiheit als Freiheit des Anderen wartet noch immer darauf, eingehalten zu werden. Was mit der politischen Philosophie der Aufklärung historisch geschieht, hat die Frankfurter Schule um Adorno und Horkheimer als deren „Dialektik“ charakterisiert. Gemeint ist damit, dass die neu entstandene 
bürgerliche Ordnung die Tendenz der Unfreiheit immer mit sich führt. Im Namen der Freiheit wird mit autoritärer Macht erzogen, normiert, kontrolliert und Recht gesprochen. Es gibt eine Freiheit, die sich staatliche Macht als Legitimationsmuster entgegenhalten lassen mussen, es gibt aber auch eine Freiheit, die im Bündnis mit staatlicher Macht durchgesetzt wird und diesen Machtanspruch verselbstständigt. Die Rechtswissenschaften sind dann nicht mehr Reflexionsmedium von Freiheit, Privatautonomie und Vertrag, sondern werden zu einem Instrument im Dienste staatlicher Ordnung umgepolt. Im 19. Jahrhundert taucht in den werdenden oder schon gewordenen Nationalstaaten die von Rechtswissenschaftlern und -politikern ersonnene Figur der „Einheit der Rechtsordnung“ auf. Recht ist Durchsetzung politischer Macht und dient deren Stabilisierung; wenn sie auch zugleich der individuellen Freiheit dient, hat man Glück gehabt. Dies ist aber nicht mehr notwendig so. Die Zuwendung der Rechtswissenschaft zur Absicherung politischer Macht wird im 19. Jahrhundert registriert und - vehement kritisiert.

Exponent solch vehementer Kritik ist Julius Hermann von Kirchmann, deutscher Richter, Politiker und Rechtsgelehrter des 19. Jahrhunderts, mit seiner Streitschrift „Über die Wertlosigkeit der Jurisprudenz als Wissenschaft“: Danach bedeutet Rechtswissenschaft vor allem Kritik und die Überwindung von Defiziten des positiven Rechts. Indes weiß schon von Kirchmann von der den Juristen eigenen blinden Reduktion des Rechts auf zufällig gewordene Regeln zu berichten - das nur positive und positivistisch wahrgenomme Recht, das die eigentliche Aufgabe der Rechtswissenschaft, einer Kultur der Freiheit Teil zu sein, verfehlt. Juristen, konstatiert von Kirchmann, seien durch das positive Gesetz zu Würmern geworden, die nur von dem faulen Holz lebten, von dem gesunden sich abwendeten, sei es nur das kranke, in dem sie nisteten und webten. Indem die Wissenschaft das Zufällige zu ihrem Gegenstand mache, werde sie selbst zur Zufälligkeit. Und dann der historisch wichtige und viel zitierte Satz: „drei berichtigende Worte des Gesetzgebers und ganze Bibliotheken werden zu Makulatur.“2

\section{Traditionelle und kritische Rechtswissenschaft}

Kritische Rechtswissenschaft, will sie also nicht bloße Makulatur sein, soll nicht weniger als die Fundamente der Freiheit im täglichen Kleinklein juristischer Detailarbeit offen legen und verteidigen. So wie Max Horkheimer und die Philosophen der Frankfurter Schule zwischen traditioneller und kritischer Theorie unterschieden, so lassen sich auch traditionelle und kritische Rechtswissenschaft auseinanderhalten. Traditionelle Rechtswissenschaft sichert den Status Quo des gerade politisch aktuellen Rechts. Sie ist funktional im Dienste und im Auftrag des politischen Systems. Abhängig vom politischen System sind ihre Gesichter vielfältig. Sie kann demokratisch sein, aber auch der Tyrannei dienen, sie schwankt zwischen Biedermaier und Brandstifter am Schreibtisch. Sie ist fokussiert auf das Recht des Nationalstaats und nur soweit international wie das positive Recht sie trägt. Eine Rechtswissenschaft in kritischer Absicht, als Teil einer Kultur der Freiheit, nimmt die Grundlagen des Rechts - seine Legitimation, seine

2 Julius Hermann von Kirchmann, Die Wertlosigkeit der Jurisprudenz als Wissenschaft, Berlin 1848 (Nachdruck Heidelberg 1988), S. 29. 
Folgewirkungen, seine Funktionsdefizite in den Blick. Rechtsphilosophie, Soziologie, Geschichte, aber auch Wirtschaftswissenschaften und die Methoden empirischer Sozialforschung gehören zu ihrem Kanon. Vor allem aber ist sie europäisch, international, rechtsvergleichend. Der Nationalstaat ist nicht ihr notwendiger Ausgangspunkt, ist vielleicht eine Frage, kann aber nicht ihre alleinige Antwort auf gesellschaftliche Problemlagen sein.

Das ist nicht nur Theorie. Traditionelle und kritische Rechtswissenschaft spiegeln sich wider - in den rechtswissenschaftlichen Fakultäten, in den Professorinnen und Professoren des Rechts und in der Konzeption juristischer Ausbildung, der sie folgen. Rechtsprofessoren, die in Vorlesungen vor 500 oder mehr Studierenden, alte Manuskripte nur vortragen, sind das abschreckende Symbol traditioneller Rechtswissenschaften. Reflexion und Kommunikation über das positive Recht findet nicht statt: Recht als starres Regelwerk, wenn auch im Detail, wird - wie Babynahrung - den Studierenden verabreicht, Fragen begreift man als Affront und das eigene Nachdenken kann sich im Examen als Nachteil erweisen. Ein solches Ausbildungssystem ist nur Schule politischer Macht, die Absicherung überkommener Ordnung, die sich im Muff des Talars geschützt glaubt - das Gegenteil einer Kultur der Freiheit.

\section{Universität, Wissenschaftsautonomie und Rechtskultur}

Die Universität ist die notwendige Vermittlerin zwischen Rechtswissenschaft und Rechtskultur. Von ihr hängt es wesentlich ab, welches Verständnis von Rechtswissenschaft geprägt wird. Sie kann - und soll - Motor einer Rechtskultur der Freiheit sein. Zur Relation von Rechtswissenschaft und Rechtskultur tritt also eine dritte Kategorie die Freiheit von Forschung und Lehre. Kritische Rechtswissenschaft, die der Kultur der Freiheit zur Entfaltung verhelfen kann, setzt zwingend die Unabhängigkeit von Forschung und Lehre voraus. Die Autonomie rechtswissenschaftlicher Forschung und Lehre mag manchem politisch unliebsam sein: genau das ist aber auch das Ziel kritischer Rechtswissenschaften, die sich vor allem in der unabhängigen Universität entfalten lassen. Kritik am Bestehenden bedeutet nämlich zugleich auch gesellschaftlichen Fortschritt. Wer ein besseres, humaneres, erfolgreicheres, demokratischeres, rechtsstaatlicheres Gesellschaftsmodell erreichen will, darf unbequeme Kritik nicht fürchten, weil die Bequemlichkeit nicht nur machtvollen und intransparenten Systemstrukturen der Politik dient, sondern auch das freiheitliche Fundament einer Gesellschaft erodieren lässt. Das Erbe der Aufklärung verlangt nach Kritik, sonst werden wir es verlieren.

Dabei ist wichtig, dass Wissenschaftsautonomie kein antiquiertes Konzept von Sozialromantikern, die sich im Elfenbeinturm teuer eingemietet haben, darstellt. Wir bilden Studierende sicher auch aus, damit sie erworbene Kenntnisse in den Dienst von Wirtschaft und Gesellschaft stellen. Das ist für Wohlstand und Wirtschaft wichtig, aber es ist nicht alles. Europäische Hochschulpolitik in ihrer Tendenz alles und jeden zu vermessen, versäumt es zuweilen, über bloßen ökonomischen Nutzen hinauszudenken. Die Aufgabe, der sich die Rechtswissenschaften an der Universität stellen müssen, ist viel komplexer. Wir leisten einen Beitrag zur freiheitlichen Entwicklung einer Europäischen Gesellschaft. Wir schützen Freiheit gegen die Mächtigen - in der Politik, in Sicherheitsapparaten, nicht zuletzt auch auf Kapitalmärkten. Dazu gilt es, Wissen, nicht 
nur Information, Kritikfähigkeit, nicht nur Kompetenzen, Selbstbewusstsein, nicht nur Dienstleistungsmentalität zu vermitteln. Klar ist, dass juristische Ausbildung an den Universitäten und die Forschung nicht losgelöst von gesellschaftlichen Problemen erfolgt. Kritik speist sich stets aus praktischer Vernunft, das heißt der möglichen Umsetzung von Prinzipien in Realität und das Hinterfragen dieser Realität im Lichte von Prinzipien. Kritische, aber eben auch moderne und innovative Juristenausbildung nimmt die Grundlagen des Rechts in den Blick, sie gewährt Einsichten in die unverrückbaren Rechtsprinzipien und sie denkt über die zu engen Grenzen des Nationalstaats hinaus. Diese Juristenausbildung kann nur glaubwürdig von Rechtslehrern und -lehrerinnen vertreten werden, denen die eigene Unabhängigkeit höchstes Gut bleibt - unverstellt von machtvollen ökonomischen und politischen Interessen Dritter.

Dies ist im Übrigen nicht elitär, aber anspruchsvoll. Ein solches Miteinander von Professoren und Studierenden und die Verzahnung von Wissenschaft, Praxis und Studium verlangt nach einer gemeinsamen Verantwortung für das Recht als Teil einer Kultur der Freiheit. Wir wollen Studierende, die sich dieser Verantwortung bewusst sind, die Fragen stellen, ihre Argumente wägen können, intrinsisch motiviert und neugierig auf internationale und europäische Herausforderungen sind. Wer nicht wagt, den eigenen Verstand im Recht zu gebrauchen, möge sein juristisches Glück andernorts versuchen.

Unsere Freiheiten sind im Moment vielleicht so sehr gefährdet wie kaum zuvor nach dem Ende des Zweiten Weltkriegs und nach der Beendigung des Ost-West-Konflikts. Eine stille, eine heimliche, eine zermürbende Erosion von Weltbürgerrechten findet vor unseren Augen statt. Es gibt nur wenige Auswege. Dazu zählen junge Juristen, deren kritischer Geist exzellent und europäisch ausgebildet wurde, dazu zählen Anwälte, die ihre Stimme erheben, dazu zählen unabhängige Rechtswissenschaftler, die beide unterstützen im Dienste einer Rechtskultur von Freiheit. 